\title{
Enhancing intra-cognitive communication between engineering designers and operators: A case study in the laser welding industry
}

\author{
C. Johansson*, J. Frostevarg **, A.F.H. Kaplan**, M. Bertoni* and K. Chirumalla* \\ * Luleå University of Technology/Division of Innovation and Design, Luleå, Sweden \\ ** Luleå University/Division of Product and Production Development, Luleå, Sweden \\ \{christian.johansson, jan.frostevarg, alexander.kaplan, marco.bertoni, koteshwar.chirumalla\}@1tu.se
}

\begin{abstract}
In manufacturing, metal parts can be joined using a laser as a welding tool, i.e. laser welding. Despite huge amount of research over the years, the process is neither sufficiently understood nor mathematically predictable. This study aims to holistically analyze the knowledge management issues occurring in laser welding. Emerging from observations and semi-structured interviews from industry and academy, the complexity and the criticalities of the process as well as the current knowledge transfers is explained and analyzed, using a knowledge lifecycle framework as a reference. Besides enhanced awareness of the limiting issues, information and knowledge visualization, e.g. knowledge maps, is identified as a key for progress in the community. The Matrix Flow Chart is suggested as an alternative descaled map of process changes.
\end{abstract}

\section{INTRODUCTION}

Industrial manufacturing processes (e.g. generating/ casting, separating, forming, joining and departing) deals with transforming raw materials into finished goods. Poor product properties from improper manufacturing can cause significant economic losses as well as product failures. Therefore, it is crucial to be able to control every aspect of the manufacturing process and thus the resulting product quality. In light of this, the fundamental processes of manufacturing are steadily becoming more knowledgebased [1]. This includes using knowledge to optimize processes, as well as capturing and transferring it to other areas where it can be used to advantage.

Any manufacturing process is essentially an Input/Output (I/O) system. The input parameters cause, during the interaction with material, a determined physical process that result in geometrical and physical output properties of the product. From a knowledge perspective, any result from a study, experiment or production can be I/O-data points, which contain information that can enable optimization of the process.

Laser welding - the joining of two parts by melting and re-solidifying their edges with a high-power laser beam is a high potential/high risk technology compared to many conventional welding techniques.

Among several welding techniques (e.g., friction stir welding, TIG, MIG/MAG, plasma), laser welding offers great depths of weld penetration at high welding speeds that results in strong welds at a high production rate, which makes it preferred both economically and from a quality perspective. However, it is also a high-risk technology both due to its high investment costs (typically $0.2-2$ million Euro) and the difficulty to balance and control a process with many heterogeneous and intertwined variables.

The welding process can be treated as an I/O system where, through processing, a certain input parameters cause a certain output $[2,3,4]$; see Figure 1(a). These mechanisms take place in complex geometrical conditions, as illustrated in Figure 1(b). Despite considerable research efforts since the 1980's, it is neither sufficiently understood $[5,6]$, nor completely measurable [7] or mathematically predictable $[8,9,10]$. Each experiment and production operation is yet another I/O data point.

The process of laser welding in manufacturing is very complex and involves many input and output parameters that are combined in a non-linear manner. To achieve good quality welds and thus a stable process, case-specific parameters must be set and tuned. There are certain parameter regions where the process is stable, however these regions are difficult to transfer to neighboring situations. This is partly due to process complexity, but also due to poor knowledge capturing and sharing. There is a problem of finding both scientific and applied knowledge about laser welding [2, 11, 12]. Engineers and operators must therefore constantly "re-invent the wheel".

What is needed is to better understand and eventually improve the knowledge capture, transfer and reuse in the intra-cognitive infocommunication [13] that occurs between the engineer or operator, who discovers and records the knowledge, and the person who needs to apply it in the next process. The medium of communication and documentation is presently inferior relative the needs of the actors.

The objective of this paper is to (i) investigate current approaches for knowledge capture, (ii) problematize the knowledge transfer process, and (iii) to elaborate on how to address the knowledge transfer challenges. 


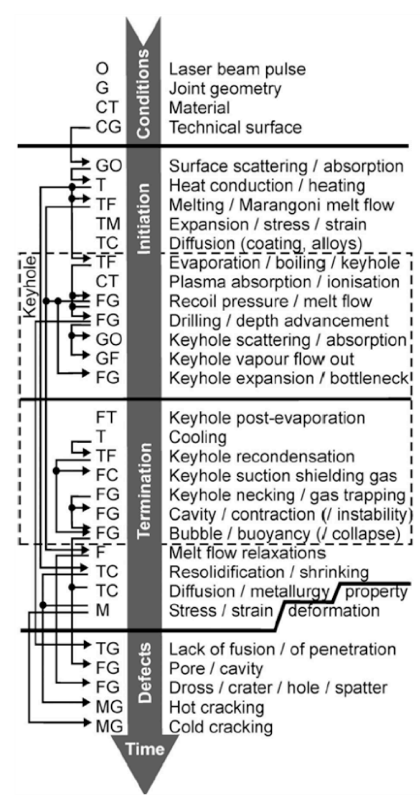

(a) (b)

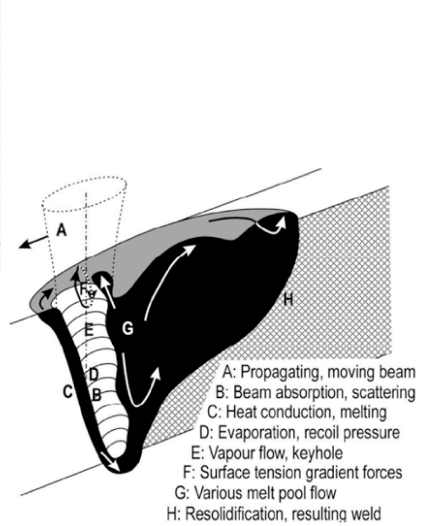

Figure 1. The complexity of the laser welding process, including laser keyhole formation, stability, molten metal flow and solidification.

\section{RESEARCH APPROACH}

The authors have adopted a qualitative research methodology, with an inductive reasoning, having its starting position in real industrial cases. Data were collected by means of observation of work methodology in laser welding. Observations have been conducted both in laboratories and in industrial settings.

In addition, the authors have conducted structured and semi-structured interviews with researchers and engineers from both academic and industrial environments. Further, discussions with users and stakeholders (e.g., engineers, operators, and managers) in industry, as well as academic experts, were a major source of information for this study. Finally, literature reviews as well as analysis of various documentations and publications in the area have served as an important complementary source of information.

\section{KNOWLEDGE MANAGEMENT}

A central objective of this study is how to capture tacit knowledge and how to turn the huge amount of data and information (produced via case studies and experiments) into systematic useful knowledge for the laser welding community. A leading technology manager at one of the leading expert groups in welding, The Welding Institute (TWI, Cambridge, UK) made the following comment:

"We even changed the catchphrase at our letter paper, to emphasize to our customers that knowledge is our core business - just we still are searching for strong solutions for how to manage it best."

Support tools for knowledge management are not universally applicable [14], but their usefulness depends on context and the stage of the knowledge. However, they are often presented in an undifferentiated way.

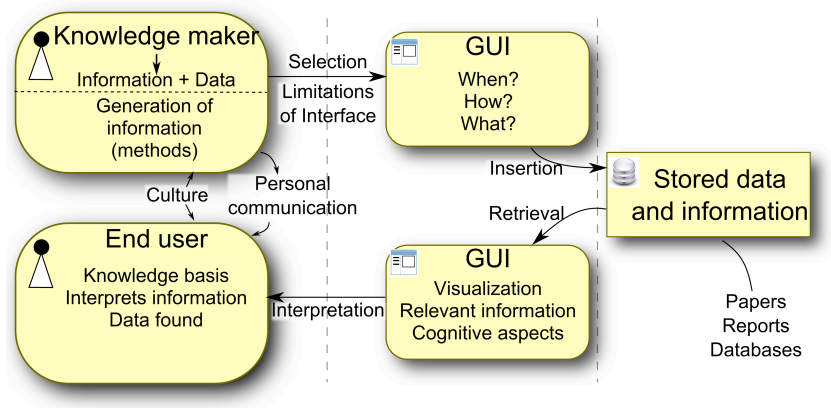

Figure 2. Knowledge path from the knowledge maker to the receiver.

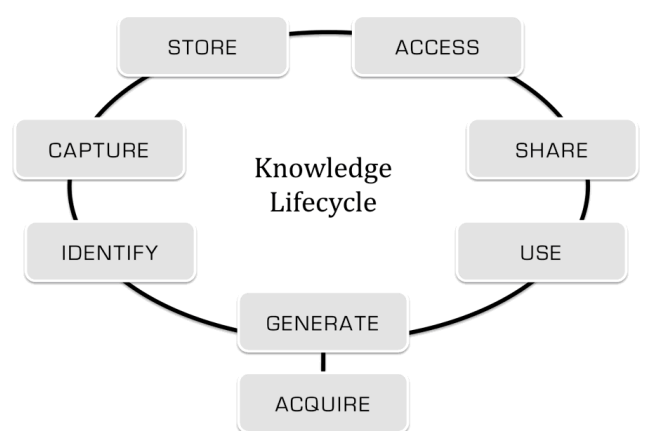

Figure 3. A generic knowledge lifecycle framework.

“...reuse often fails because knowledge is not captured; it is captured out of context, rendering it not reusable; or there are no formal mechanisms for finding and retrieving reusable knowledge." [15]

A major focus for many companies' $\mathrm{KM}$ efforts is to assist and motivate employees to share knowledge with colleagues and to contribute to their companies' shared knowledge repositories [16].

\section{A. Knowledge Lifecycle}

When searching for knowledge, the recipient may be guided or limited by inherent knowledge and interpretation of the thematic subject. How the content is structured and visualized may increase the recipient's navigation and understanding. The selection of knowledge depends largely on the systems used to retrieve information from stored devices and its inherent structure. The path from knowledge maker to an eventual recipient (see Figure 2) goes well in hand with the generic knowledge lifecycle [17]. Figure 3 shows the framework that foresees seven main phases: Acquire/Generate (connected), Identify, Capture, Store, Access, Share, Use (Learn).

\section{KNOWLEDGE UTILIZATION IN LASER WELDING}

Once specified, a defined output (i.e., product quality) of the process must be achieved by choosing a suitable input (e.g., system parameters, work piece conditions, and tolerances). This knowledge either directly exists or must be generated, which is expensive.

Successfully reusing knowledge from one welding case to another is hindered by difficulties of in understanding the conditions of when two cases are "similar" (in the same parameter window) and discerning case-specific and generic knowledge elements (i.e. information captured out 
of context). When developing high-quality welding operations, experience is an important element.

When engineers develop a new weld operation, they limit the choice of parameters to the preferred and manageable ones. This leaves the rest as uncertainties, as it is unclear how these parameters mutually affect each other. Even when considering only the 20 most obvious parameters, it is unfeasible to systematically test all possible combinations, as the tests are too time consuming and expensive. Some root-cause relationships may be well known, but the majority remains unknown.

In addition, there are also uncontrollable variables, such as absorption rates, laser beam power stability, scattering of the beam in the vapor plane, and machine movement accuracy. Even if all parameters are controlled and stable, they still behave in a non-linear manner.

To find parameter windows with acceptable quality, the most common approaches are either analytical trial-byerror or design of experiments. The disadvantage of the first method is that it does not generate so much valuable data, whereas the second enables to interpolate mathematically between isolated studies, if there are monotonic trends. However, they are only locally valid, and thus unsatisfactory, for laser welding.

Multivariate analysis, such as parallel coordinates, is an interesting approach, but due to the lack of complete datasets [18].

Except for a few theoretical studies, all research is case studies, which are documented by describing conditions, parameters and results. This generates large quantities of reports and publications, which are difficult to generalize beyond the specific case. Additionally, only a select part is analyzed deeper and illustrated or formalized. The rest (which is the most) of the results remain stored in an unstructured manner or are even deleted [19]. Apart from finding the relevant knowledge at all, another issue is to find the relevant information when it is needed. There is potential in putting efforts in structuring the tacit knowledge and codifying the codifiable tacit knowledge, but the benefits are currently outweighed by the high costs compared to other visible investment possibilities and therefore has low investment priority [4].

\section{FINDINGS OF THE STUDY}

In order to extract the requirements for a solution, able to better support knowledge codification and exchange, the knowledge management process of laser welding cases has been analysed using a knowledge lifecycle framework.

\section{A. Generate and Use}

When studying a new welding case, searching for similar situations, best practices or guidelines is time consuming and may not render useful for the case at hand.

New knowledge is mainly generated from conducting experiments, despite being expensive, time consuming and difficult to setup.

Knowledge management activities are, therefore, mostly case driven, where neither broad mapping nor generalization is conducted $[20,21,22]$.
Finding 1: There is a need for methods and tools that improves visualization and interpolation of case study results.

\section{B. Access and Share}

The scarce existing knowledge and information is stored in a multitude of different formats (e.g., reports, journals, notes) and systems, which often are difficult to access.

Also, the study has shown that much knowledge is exchanged by word-of-mouth, which implies that people need to know who to address with a problem. Methods and tools allowing users to locate the right experts are needed.

Finding 2: Methods and tools are needed to support users in classifying the knowledge they possess - in a way so that others can reuse it. This includes being able to position new knowledge elements relative to pre-existing knowledge elements.

Finding 3: Methods and tools are needed to access knowledge owners within and across organizations.

\section{Store}

Predefined boundaries and storage constraints of the medium limits the way results can be expressed, thus leading to omission of details. What is captured is often governed by ingrained rules and standards based on conventions in databases, reports and journals.

To move towards generalization of results, there is a need for a system that is more flexible in structure and content. Stakeholders need to be able to interact and attach knowledge elements, such as comments (e.g., recommendations and pitfalls), ratings, illustrations and other extensions.

Finding 4: Methods and tools that are more customizable and "media rich" [23] are needed to store contextual information useful for generalization.

\section{Identify and Capture}

The study has shown that a challenge is how to capture the unstructured, tacit knowledge of experts. As one engineer at Trumpf AG (the world's largest high-power laser manufacturer) says:

"We have developed a comprehensive database and successfully use design-of-experiments for interpolation, but it only works in certain areas known by an expert, thus we need this accompanying tacit knowledge."

From an industrial perspective, a major problem is the documentation of lessons learned related to operation execution. A series of data points might contain useful information (e.g., trends) for other data points (e.g., how to suppress a specific defect). This information, and the range of validity, is not trivial to extract, and is thus a result of a long learning-by-doing process. It is ingrained in experience and rarely formalized and shared with others.

The authors have also observed that personal interaction and verbal (informal) communication is believed to be a faster and more reliable way to gather needed information. 
However, this approach is limited in the sense that finding the right expert can be difficult.

Finding 5: Methods and tools are needed lower the threshold for capturing tacit and unstructured knowledge in the laser welding process, as well as the rational and lessons learned acquired during planning, testing and execution.

\section{MATRIX FLOW CHARTS}

As a remedy for the findings in the previous section, the authors suggest a pioneer approach that is under development [24], the Matrix Flow Charts (MFC) (see Figure 4). The intention is to record and display knowledge at a level of abstraction that matches the issues in the laser welding process. The MFC visualizes qualitative parameter trends from laser welding experiments instead of the traditional way of listing their numerical data relations.

To read the chart, start from the titles above the vertical lines (i.e., input parameters), follow the vertical lines down to an intersection with a black dot, which depicts that there is a correlation at this pint with the output that is at the end of the corresponding horizontal line (i.e., result). Reversed, by identifying a certain output, the MFC makes it possible to backtrack the welding process up to its inputs and then to identify the proper actions to cope with the problem. To suppress the defect, change the corresponding parameter into a state free from the defect. For instance, increasing the inclination angle or the welding speed can eliminate undercut or root sagging. Both parameters have defects in one state, but not in the other.

The aim is that the MFC should be as "lightweight" as possible. Detailed information is not disclosed in this representation, because the idea is to support many different applications and parameters regimes. Specific parameter values change with every new case, but trends captured in the MFC may still be valid and patterns of trends could be detected by these means.

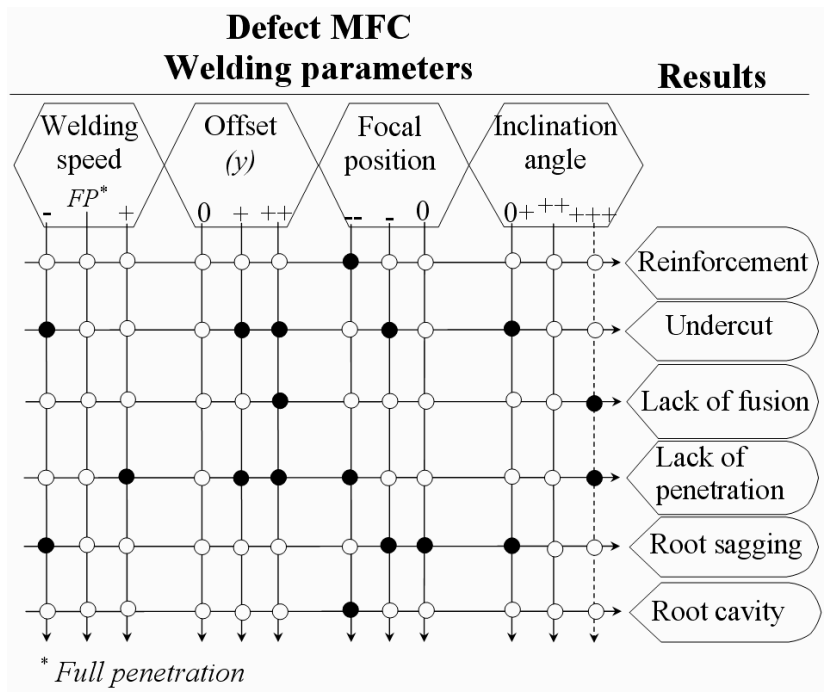

Figure 4. The developed solution approach called the Matrix Flow Chart, MFC.
The MFC displays correlations between input parameters and outputs in a single environment. Further development of the method is to embed and connect different (and, to some extent, non conventional) knowledge sources to the knowledge base, so that existing documentation, notes or pictures can be attached to each intersection point and be accessed by experts to clarify and explain the I/O-relation.

The main benefits of the approach relate both to the possibility of lowering the threshold for capturing essential information of the laser welding operations, and of enhancing the capability to overview the knowledge base, as a means to make easier for experts to decide if to look for additional information on existing lessons learned or to go for a new case study.

\section{DISCUSSION}

Laser welding, in spite of its high level of automation, is an activity still treated, to a large extent, as a skill or a craft, mainly because of the complex relationship between input properties and output effects (and defects). Such knowledge and skills, however, mostly remains tacit and hidden across the organization, resulting in situations where knowledge is not reused when designing a new case study. Several problems have been identified while analysing the knowledge sharing process with the aid of the knowledge lifecycle framework. The existing knowledge support tools perform poorly and most knowledge remain tacit or scattered across various notes.

There is a need for systematic collection (mapping) of data and information, along with generalization and limits for each rule in order to facilitate the search navigation through it.

The MFC, which has been presented in this paper, can be seen as a communication device (see Figure 5) and will therefore enable and improve the intra-cognitive infocommunications between two actors [13]. Essentially it will work as an interface between them to overcome the challenges both in terms of communicating and sharing knowledge.

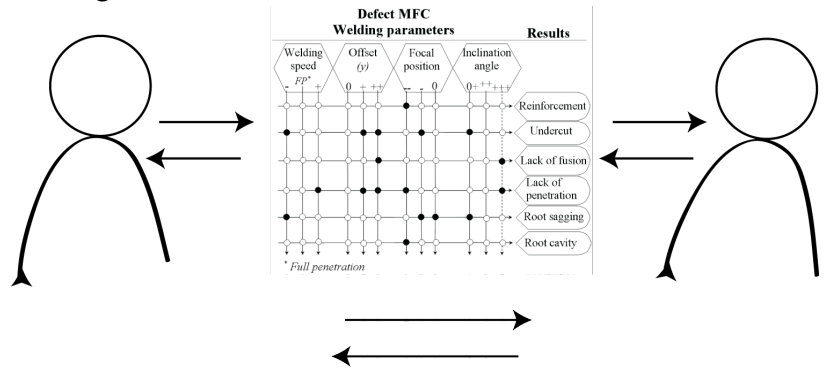

Figure 5. Using the MFC to enhance intra-cognitive infocommunication, adapted from [13]. 


\section{CONCLUSIONS}

The paper has holistically explored the reasons for the poor knowledge sharing performances in laser welding in the manufacturing domain, identifying several critical issues at different phases of the knowledge lifecycle.

In spite of the huge amount of knowledge resources available, large reinvention efforts takes place, due to several intertwined factors. Industry often prefers case studies, generating isolated data-points, while the desired transferable knowledge is hardly generated, as requiring long-term basic research. The formulation of trends and categories by capturing tacit knowledge and by identifying trends from the case studies would be a solution.

In terms of documenting, there is a need for an approach that is easy to work with and not so cumbersome, possibly sacrificing extensive detail for an improved overview of the whole knowledge base. In addition to this, there is also a need to capture the tacit knowledge of the experts and make use of verbal knowledge sharing.

The paper has presented the MFC as an approach as an aid to gather knowledge elements in a visual map. There might be might potential in further expanding the scope of the approach close links to media rich information and knowledge sources, e.g. lessons learned.

\section{ACKNOWLEDGMENT}

The authors are grateful for funding by VINNOVA The Swedish Governmental Agency for Innovation Systems (project LOST, no. 2006-00563) and to the Research Council of Norrbotten (project no. NoFo 09004) for funding the research.

\section{REFERENCES}

[1] EU Commission, Manufuture High Level Group Report, 2004.

[2] W. W. Duley, Laser Welding. New York, NY: WileyInderscience, 1998

[3] A. F. H. Kaplan, "A model of deep penetration laser welding based on calculation of the keyhole profile," J. Phys. D. Appl. Phys., vol. 27, no. 8, pp. 1805-1814, September 1994.

[4] B. Ribic, T. A. Palmer, and T. DebRoy, "Problems and issues in laser-arc hybrid welding," Int. Mater. Rev., vol. 54, pp. 223-244, July 1999.

[5] A. F. H. Kaplan, "Influence of the beam profile formulation when modeling fiber guided laser welding," J. Laser Appl., vol. 23, no. 4, pp. 1-9, March 2011.

[6] S. Moore, A. Conneely, E. Stenzel, and E. Murphy, "Investigation of the application of process analytical technology for a laser welding process in medical device manufacturing," Physics Procedia, vol. 12, pp. 656-665, April 2011.
[7] R. Olsson, J. Eriksson, J. Powell, and A. V. Langtry, "Challenges to the interpretation of the electromagnetic feedback from laser welding," Opt. Laser Eng., vol. 49, no. 2, pp. 188-194, February 2011.

[8] A. Belhadj, J. Bessrour, J.-E. Masse, et al. "Finite element simulation of magnesium alloys laser beam welding," J. Mater. Process Tech., vol. 210, no. 9, pp. 1131-1137, June 2010.

[9] H. Koch, K.-H. Leitz, A. Otto, and M. Schmidt, "Laser deep penetration welding simulation based on a wavelength dependent absorption model," Physics Procedia, vol. 5, pp.309-315, August 2010.

[10] W.I. Cho, S.-J. Na, C. Thomy, and F. Vollertsen, "Numerical simulation of molten pool dynamics in high power disk laser welding," J. Mater. Proc. Tech., vol. 212, no. 1, pp. 262-275, January 2012.

[11] U. Gratzke, P. D. Kapadia, and J. Dowden, "Heat conduction in high-speed laser welding," J. Phys. D. Appl. Phys., vol. 24, no. 12, pp- 2125-2134, December 1991.

[12] J. F. Lancaster, The physics of welding. Oxford, UK: Pergamon Press Ltd., 1984.

[13] P. Baranyi, and Á. Csapó, "Definition and synergies of cognitive infocommunications," Acta Polytechnica Hungarica, pp 67-83, vol. 9, no. 1, 2012.

[14] J. Birkinshaw, and T. Sheehan, "Managing the knowledge lifecycle," MIT Sloan Manage. Rev., pp. 75-83, Fall 2002.

[15] R. Fruchter, and P. Demian, "CoMem: designing an interaction experience for reuse of rich contextual knowledge from a corporate memory," Ai Edam, vol. 16, no. 3, pp. 127-147, June 2002.

[16] C. Small, and A. Sage, "Knowledge management and knowledge sharing: A review," Info. Know. Sys. Manage., vol.5, pp. 153169, November 2006.

[17] P. Nuzzo, and F. Lockwood, Knowledge enabled solution components state of the art. VIVACE Project report.

[18] A. Inselberg, and B. Dimsdale, "Parallel coordinates: a tool for visualizing multi-dimensional geometry," VIS'90, vol. 1, pp. $361-378,1990$.

[19] S. Bell, Lean enterprise systems: Using IT for continuous improvement. Hoboken, NJ: John Wiley \& Sons, 2006.

[20] W. Fricke, "Fatigue analysis of welded joints: state of development," Mat. Struct., vol. 16, no. 3, pp. 185-200, May 2003.

[21] T. Jokinen, T. Vihervä, H. Riikonen, and V. Kujanpää, "Welding of ship structural steel A36 using a Nd: YAG laser and gas-metal arc welding," J. Laser Appl., vol. 12, no. 5, pp. 185-188, June 2000.

[22] S. K. Panda, N. Sreenivasan, M. L. Kuntz, and Y. Zhou, "Numerical simulations and experimental results of tensile test behavior of laser butt welded DP980 steels," J. Eng. Mat. Tech., vol. 130, no.4, pp. 554-571, May 1986.

[23] R. L. Daft, and R. H. Lengel, "Organizational information requirements, media richness and structural design," Manage. Sci., vol. 32, no. 5, pp. 554 - 571, May 1986.

[24] J. Karlsson, and A. F. H. Kaplan, "Analysis of the surface geometry of a fibre laser welding case study, utilizing a Matrix Flow Chart," Appl. Surf. Sci., vol. 257, pp. 4113-4122, February 2011. 